\title{
Detection of high energy electromagnetic and hadron components of air-shower cores in the new hybrid experiment "Pamir-XXI"
}

\author{
M. Tamada ${ }^{1, \text { a }}$, N. Inoue ${ }^{2}$, A. Misaki ${ }^{2}$, and A. Ohsawa ${ }^{3}$ \\ 1 Faculty of Science and Engineering, Kinki University, Higashi-Osaka, Japan \\ 2 Faculty of Science, Saitama University, Saitama, Japan \\ ${ }^{3}$ Institute for Cosmic Ray Research, University of Tokyo, Kashiwa, Japan
}

\begin{abstract}
In the Chacaltaya hybrid experiment we have shown that the observed characteristics of the events accompanying atmospheric families (a bundle of high energy particles in the air-shower core) can not be well described by current simulations. The atmospheric families detected so far by emulsion chambers (sandwiches of X-ray films and lead plates) are key ingredients in the analysis. But the number of analyzed events with atmospheric family is still small due to the limited size of the experiment. Now a new very large hybrid experiment "PAMIR-XXI" is proposed to be constructed at the Pamirs. The notable feature of the experiment is to construct large hadron calorimeters at the center of air-shower arrays to study the air-shower core in detail. We study the possibility to analyze high energy air-shower cores in the "Pamir-XXI" experiment by using the burst density of scintillation detectors instead of using the family data of emulsion chambers. It is shown that the unusual characteristics of the events observed by the Chacaltaya hybrid experiment can be well seen in the hybrid experiment "PAMIR-XXI" too.
\end{abstract}

\section{Introduction}

In the Chacaltaya hybrid experiment[1-3] we have studied in detail high-energy air-shower cores by observing three components in the air-showers, i.e., air-shower size, burstsize (hadron-component) and accompanied family energy (high energy e, $\gamma$-component). We have shown that the characteristics of the observed events, in the energy region of primary particles $E_{0}=10^{15} \sim 10^{17} \mathrm{eV}$, can not be fully explained by simply changing the chemical composition of primary particles, though many experimental groups claim that the fraction of heavy primaries increases rapidly beyond the "knee" and that the change of the chemical composition of primary cosmic-rays is an origin of the "knee" and well explain the observed characteristics of the air-showers[4-7].

In the Chacaltaya hybrid experiment, the observed characteristics of each component itself look like they are well described by simulations, but if the events are divided into two groups, with family and without family, the simulations fail to describe the characteristics of both groups of data. Thus the information on the families is a key ingredient for understanding the characteristics of cosmic-ray interactions in the energy range of $10^{15} \sim$ $10^{17} \mathrm{eV}$. To date atmospheric families are detected by emulsion chambers, but the measurement and analysis of the family data using emulsion chambers is very timeconsuming. Furthermore there are some difficulties in connecting the air-shower to the accompanied family, because the latter has no time information. So the number of observed events with atmospheric family is still very

a e-mail: msnbtamada@gmail.com small and it is necessary to get at least ten times more family events to make the argument clear. It is preferable to use scintillation counters instead of X-ray films at a suitable depth of the lead plates to detect highenergy atmospheric families in future large-scale hybrid experiment. Here we study the possibility to analyze high energy air-shower cores by using scintillation detectors instead of using emulsion chambers for detecting high energy atmospheric families in the new comprehensive EAS experiment "Pamir-XXI" at the Pamir [8].

The experimental data detected by the Chacaltaya hybrid experiment are converted into "experimental data" expected to be detected in the "PAMIR-XXI" experiment through detailed detector simulations of both experiments and then the characteristics of the "expected experimental data" are compared with those of simulated data of airshower cores.

\section{Results of the hybrid experiment at Mt. Chacaltaya}

\subsection{Chacaltaya hybrid experiment}

The air-shower array covers a circular area within a radius about $50 \mathrm{~m}$ by 35 plastic scintillation detectors to measure the lateral distribution of the electron density of the air-showers. In the center of the air-shower array, 32 blocks of emulsion chambers $\left(0.25 \mathrm{~m}^{2}\right.$ each $)$ are installed. Each block of the emulsion chamber consists of 30 lead plates each of $0.5 \mathrm{~cm}$ thickness and 14 sensitive layers of X-ray film which are inserted after every $1 \mathrm{~cm}$ lead. Burst detectors with $5 \mathrm{~cm}$ thick plastic scintillator are installed

(C) The Authors, published by EDP Sciences. This is an Open Access article distributed under the terms of the Creative Commons Attribution License 4.0 (http://creativecommons.org/licenses/by/4.0/). 


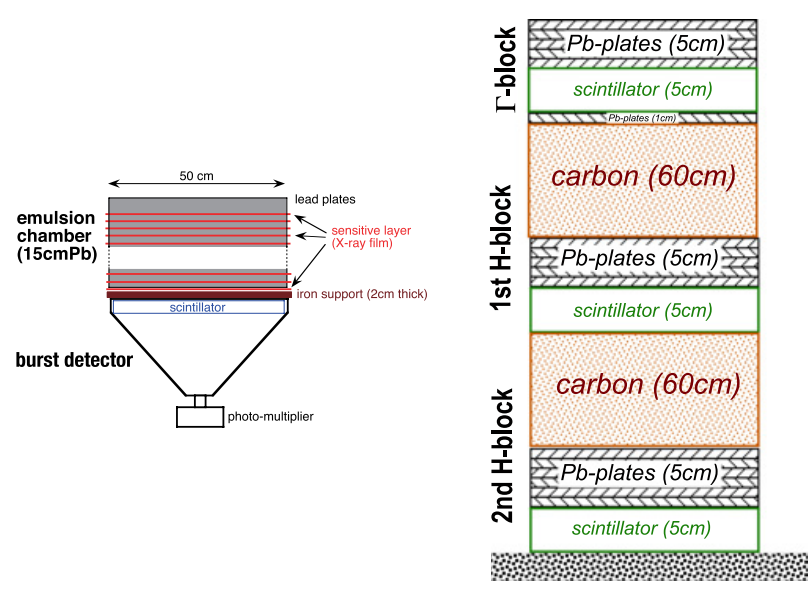

Figure 1. Structure of the hadron-calorimeter of the Chacaltaya hybrid experiment (left) and that assumed for the PAMIR-XXI experiment (right).

underneath the respective blocks of the emulsion chamber (see Fig. 1). A $2 \mathrm{~cm}$ thick iron support is inserted between the emulsion chamber and the hadron calorimeter. The total area of the emulsion chambers is $8 \mathrm{~m}^{2}$. Details of the Chacaltaya hybrid experiment are described in Refs. [1,2].

In the Chacaltaya hybrid experiments, we can obtain the air-shower size, $N_{e}$, from the air-shower array data, burst-density (the number of charged particles in $50 \times$ $\left.50 \mathrm{~cm}^{2}\right), n_{b}$, which are closely connected to the hadron component in the air-shower, from the hadron calorimeter (burst detector) and the energy and geometrical position of individual high energy electromagnetic particles of atmospheric families from the emulsion chamber. Correlations between air-showers and accompanying families were studied by comparing experimental and simulated data.

\subsection{Main results}

We have analyzed 1,037 events with maximum burst density $n_{b}^{\max } \geq 10^{4}$ among 32 blocks of burst detectors and air-shower size $N_{e} \geq 10^{6}$ in the $\sim 40 \mathrm{~m}^{2}$ year exposure of hadron calorimeters. Among them 62 events are accompanied with a family of $\Sigma E_{\gamma} \geq 10 \mathrm{TeV}$ [3,9].

Figure 2 shows air-shower size, $N_{e}$, dependence of the maximum burst-density, $n_{b}^{\max }$, of the event for all the events with $n_{b}^{\max } \geq 10^{4}$ and for those which accompany an atmospheric family of $\Sigma E_{\gamma} \geq 10 \mathrm{TeV}$. The experimental data are compared with those of simulated data by CORSIKA [10] using QGSJET01 [11]. The experimental data look like they are well described by the model calculations if we use all the events with $n_{b}^{\max } \geq 10^{4}$, but if we pick only the events accompanied by a family, the simulations fail to describe the experimental data, i.e., $n_{b}^{\max }$ of the experimental data are systematically larger than that of simulations.

Figure 3 shows a correlation diagram between $n_{b}^{\max }$ and accompanying family energy $\Sigma E_{\gamma}$. The experimental data are compared with those of simulated data. As naturally expected, $n_{b}^{\max }$ is roughly proportional to the family energy in the simulated data irrespective of the primary particles though $n_{b}^{\max }$ of the events coming from iron-primaries is larger than that from proton-primaries. We can see much of the experimental data is located
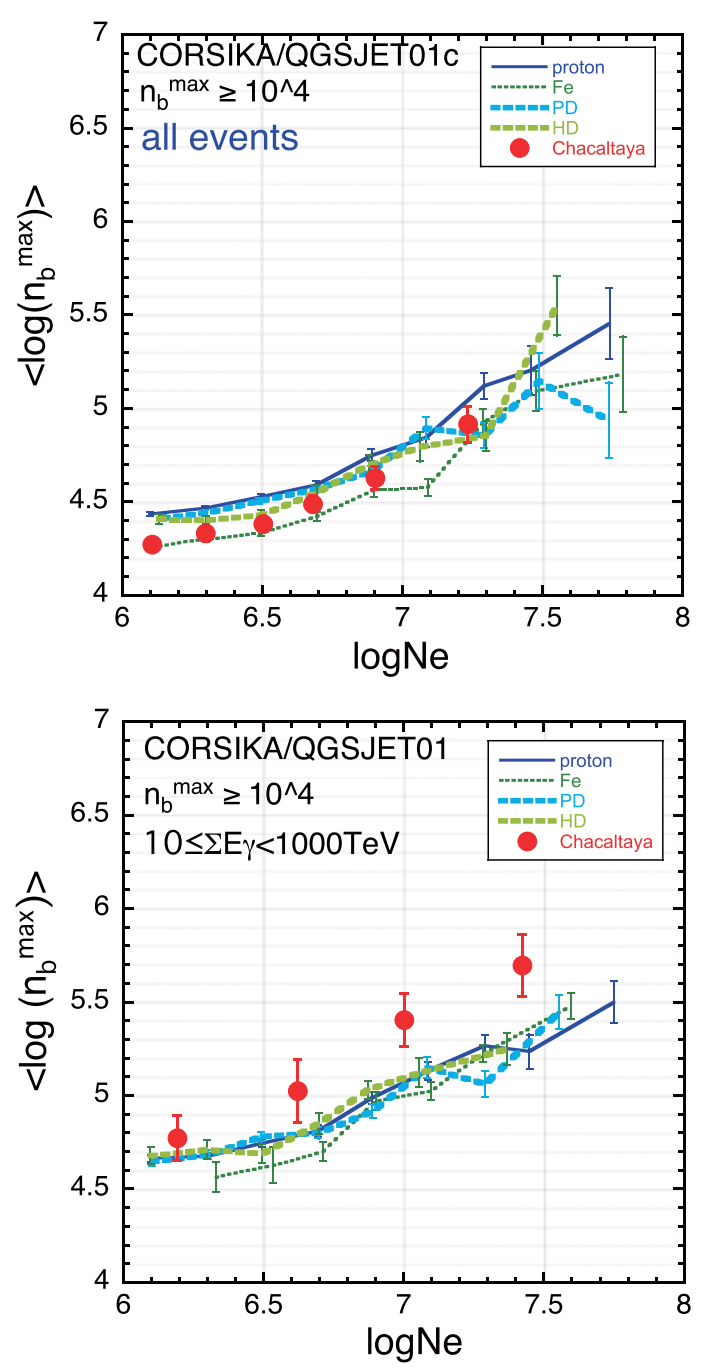

Figure 2. Shower size, $N_{e}$, dependence on maximum burst density, $n_{b}^{\max }$, for the total observed events (upper) and for those accompanying family with energy $\Sigma E_{\gamma} \geq 10 \mathrm{TeV}$ (lower) in the Chacaltaya hybrid experiment. Lines are those for the simulated events by CORSIKA/QGSJET01 with different primary particles.

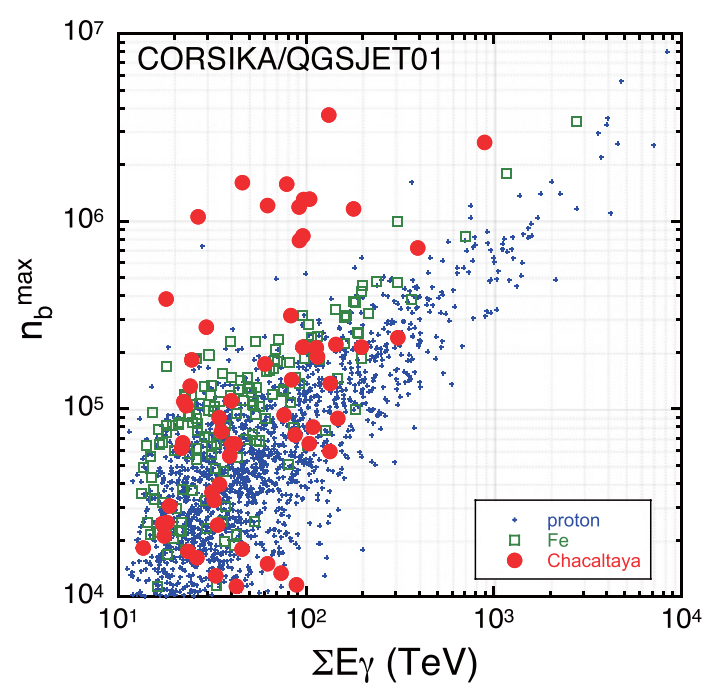

Figure 3. Correlation diagram between $n_{b}^{\max }$ and family energy $\Sigma E_{\gamma}$ of the events with atmospheric families in air-showers of $10^{6} \leq N_{e}<10^{8}$. Large closed circles are those for the Chacaltaya data. Small crosses are for simulated data of proton primaries and squares for iron primaries. 
far from the simulated events in the diagram, i.e., there exist events which have larger burst-density, $n_{b}^{\max }>\sim 10^{6}$, though the accompanied family energy is not so large.

\section{The project of "PAMIR-XXI" experiment}

A new hybrid experiment "PAMIR-XXI" at the Pamir mountains $(\sim 4,300 \mathrm{~m}$ above sea level) is proposed to study the primary cosmic-ray spectrum and chemical composition in $10^{15} \sim 10^{18} \mathrm{eV}$ as well as nuclear interactions at super high energy. The extraordinary characteristics of the experiment is to construct very large lead-carbon calorimeters $\left(192 \mathrm{~m}^{2}\right.$ with thickness of $\sim 3.5 \lambda_{\text {int }}$ ) to study in detail the air-shower core at the center of the dense EAS array $\left(80 \times 80 \mathrm{~m}^{2}\right)$ surrounded by sparse EAS array $\left(1 \times 1 \mathrm{~km}^{2}\right)$. Details of the experiment are described in Ref. [8].

\section{Simulations for the "PAMIR-XXI" experiment}

\subsection{Air-showers}

To generate extensive air-showers we use the CORSIKA simulation code (version 74005) [10] employing the QGSJET model (QGSJET01d) [11] for cosmic-ray nuclear interactions. Primary particles of $E_{0} \geq 10^{15} \mathrm{eV}$ are sampled respectively from the power law energy spectrum of integral power index -1.8 , for pure protons and pure iron nuclei. The thinning energy in the calculations in air-showers is fixed to be $10 \mathrm{GeV}$. Shower sizes, $N_{e}$, at Chacaltaya and the Pamir are calculated by using EGS4. Here we set the low energy cut-off of the particles to be $E_{c u t}=0.3 \mathrm{GeV}$ for hadrons and muons and $E_{\text {cut }}=0.001 \mathrm{GeV}$ for electromagnetic particles.

In order to compare with the Chacaltaya hybrid experiment, we assume 32 hadron calorimeters of the "Pamir-type", each of which has an area $50 \times 50 \mathrm{~cm}^{2}$, are located similar to those of the Chacaltaya hybrid experiment $[1,2]$.

\subsection{Calculation of the burst-density in $\Gamma$-block and hadron-block of the calorimeter}

Here we consider calorimeters with two carbon layers of $60 \mathrm{~cm}$ thick (see Fig. 1) for the "Pamir-XXI" experiment ${ }^{1}$. The upper part, $\Gamma$-block, of the calorimeter which consists of the emulsion chamber of $6 \mathrm{~cm}$ $\mathrm{Pb}$ and scintillator of $5 \mathrm{~cm}$ thickness, mainly detect electromagnetic cascade showers of the e, $\gamma$-component in the air-shower core and the lower part, hadronblocks each of which consists of an emulsion chamber of $5 \mathrm{~cm} \mathrm{~Pb}$ and scintillator of $5 \mathrm{~cm}$ thickness under a $60 \mathrm{~cm}$ thick carbon layer mainly detect hadronic showers produced by local nuclear interactions of the hadron component in the lead plates and carbon layer. We use GEANT4 code (version 4.10.0) [12] with the QGSP model for hadronic interactions to calculate the burst-density.

\footnotetext{
${ }^{1}$ There are 3rd hadron-block with carbon layer and 4th hadronblock with thick lead layer under the 2nd hadron-block in the original design of the calorimeter of the "PAMIR-XXI" [8].
}

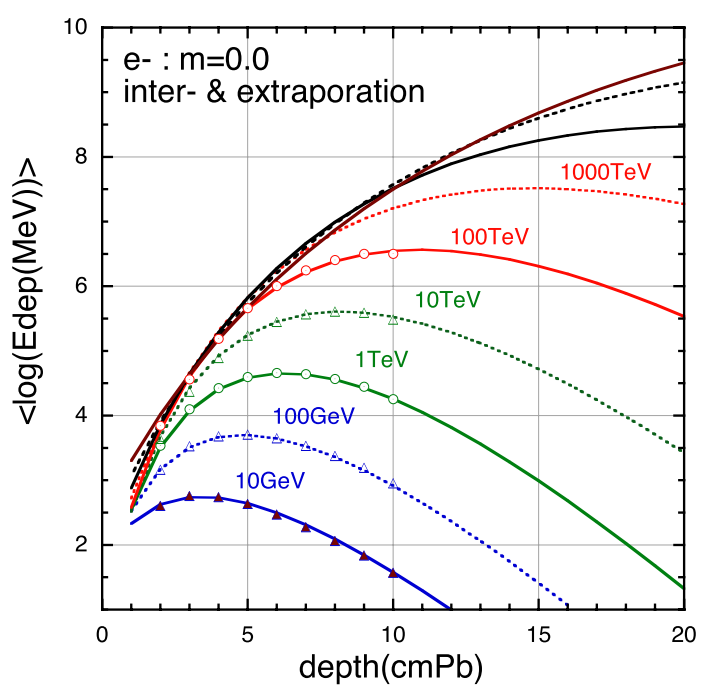

Figure 4. Average cascade transition on energy deposit in the $5 \mathrm{~cm}$ thick plastic scintillators for electrons with vertical incidence and various energies. Curves are those of approximated numerical functions based on the NKG function.

We calculate nuclear and electromagnetic cascades in the calorimeter and the energy deposit in the $5 \mathrm{~cm}$ thick plastic scintillator, located in the $\Gamma$-block and two hadron-blocks, is calculated for incident hadrons (pions, protons, neutrons and kaons) as well as for muons and $e, \gamma$ with 5 different energies of $10 \mathrm{GeV}, 100 \mathrm{GeV}, 1 \mathrm{TeV}$, $10 \mathrm{TeV}$ and $100 \mathrm{TeV}$, and 3 different zenith tangent of arrival direction, $\tan \theta=0.0,0.5,1.0$.

For the $\Gamma$-block, the energy deposit is calculated assuming the $5 \mathrm{~cm}$ thick plastic scintillator is located at various depths, under $2 \mathrm{~cm} \mathrm{~Pb}, 3 \mathrm{~cm} \mathrm{~Pb}$, and so on, in order to find the best position of the scintillation counter. Figure 4 shows examples of the average transition on energy deposit in the scintillator for electrons with vertical incidence. For the hadron-block, the energy deposit is calculated assuming the scintillator is located under $5 \mathrm{~cm}$ $\mathrm{Pb}$. In Fig. 5 we show examples of distributions of the energy deposit in the scintillator of the 2 nd hadron-block for pions with vertical incidence. The distribution of the energy deposit obtained by GEANT4 simulations are approximated by numerical functions. The distributions of the energy deposit obtained by sampling from these numerical functions which approximate GEANT4 results are shown in the figure. The distributions agree well with those obtained by full simulations using GEANT4.

In order to save computing time, the energy deposit is sampled from the average transition curves approximated based on the NKG function (see curves in Fig. 4) for the $\Gamma$-block and from the approximated numerical functions for the hadron-block (see curves in Fig. 5) for every particle incident upon the hadron calorimeter. This is converted into a particle number using average energy loss $(=10 \mathrm{MeV})$ of a single muon in the $5 \mathrm{~cm}$ thick plastic scintillator. Finally we get the burst-density, $n_{b}$, defined by the number of particles per $0.25 \mathrm{~m}^{2}$, in each block of 32 hadron calorimeters ${ }^{2}$. ${ }^{2}$ High energy e, $\gamma$ particles give energy deposits in the
scintillation counter of the hadron block. The contribution from
e, $\gamma$ particles to the burst-density is less than $\sim 5 \%$ in the 2 nd 


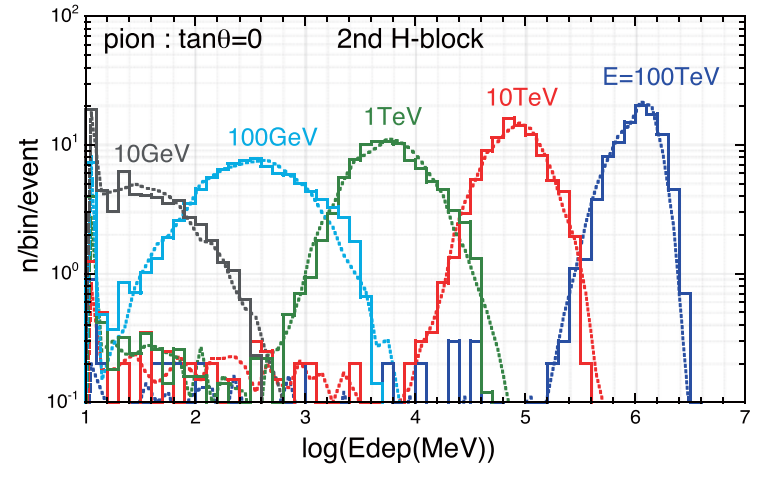

Figure 5. Distribution of the energy deposit, $E_{\text {dep }}$, in the $5 \mathrm{~cm}$ thick plastic scintillator located under $5 \mathrm{~cm} \mathrm{~Pb}$ in the 2nd hadron-block of the calorimeter for pions with vertical incidence. Histograms are results obtained by the GEANT4 simulation and dashed-curves are those obtained by sampling from approximated numerical functions.
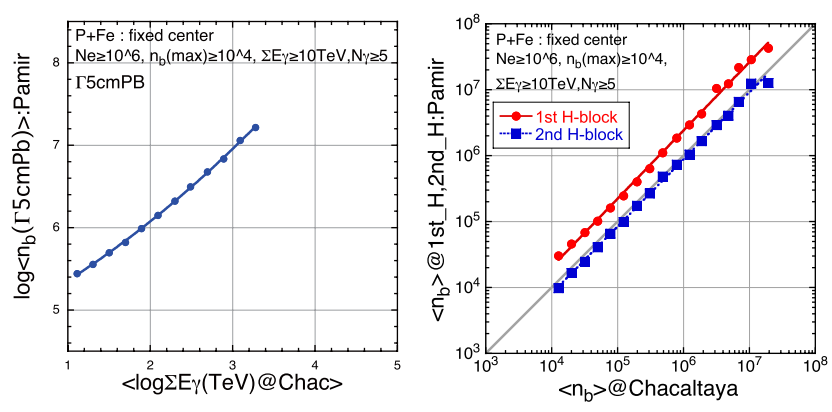

Figure 6. The average relationship between the family energy, $\Sigma E_{\gamma}$, given by the Chacaltaya hybrid chamber and burst density in the scintillator of the $\Gamma$-block, $n_{b}(\Gamma)$, of the "PAMIR-XXI" experiment (left), and that between the burst density given by the Chacaltaya burst detector, $n_{b}(\mathrm{Chac})$ and that by the hadron-block, $n_{b}\left(1 \mathrm{st} \_\mathrm{H}\right)$, and $n_{b}\left(2 \mathrm{nd} \_\mathrm{H}\right)$, of the PAMIR-XXI detectors (right).

\section{Expected results in "PAMIR-XXI"}

\subsection{Estimation of the expected experimental data}

Let's consider how the events detected by the Chacaltaya hybrid experiment can be observed by the detectors of "PAMIR-XXI" experiment. Suppose the one and the same simulated air-shower event is detected by both the Chacaltaya hybrid experiment and by the"PAMIR-XXI" experiment. Through the detector simulations for both experiments $^{3}$, we can compare the family energy, $\Sigma E_{\gamma}$, observed by the Chacaltaya emulsion chamber and the burst density in the scintillator of the $\Gamma$-block, $n_{b}(\Gamma)$, of the "PAMIR-XXI" experiment. Then we get an average relationship between $\Sigma E_{\gamma}$ at Chacaltaya and $n_{b}(\Gamma)$ at the Pamir. The relationship between $\Sigma E_{\gamma}$ and the burst density under $5 \mathrm{~cm} \mathrm{~Pb}$ in the $\Gamma$-block, $n_{b}(\Gamma 5 \mathrm{~cm} \mathrm{~Pb})$, is shown in Fig. 6. In the same way as above, we can get an average relationship between the burst density given

hadron-block but is $30 \sim 40 \%$, in average, in the 1 st hadronblock.

${ }^{3}$ Details of detector simulations of the Chacaltaya hybrid experiment is described in Ref. [13].
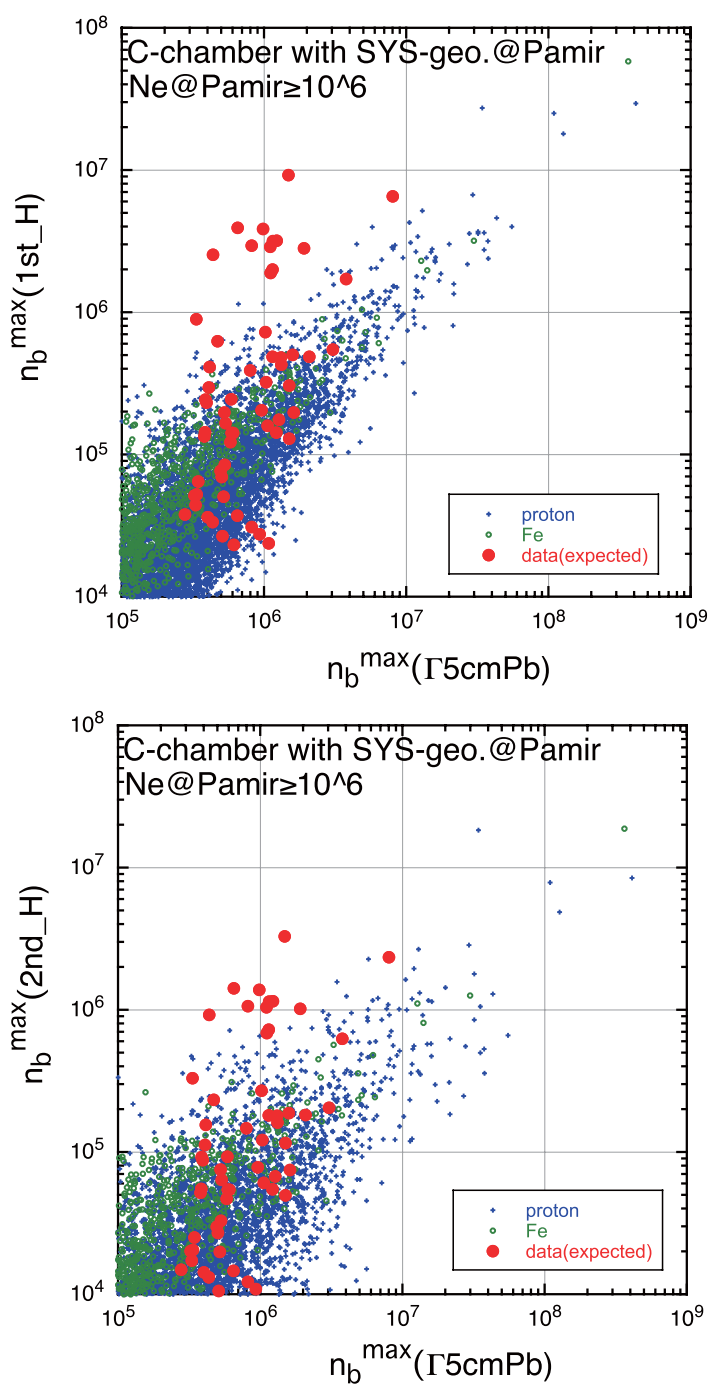

Figure 7. Correlation diagram between the maximum burst density at the 1 st hadron-block, $n_{b}^{\max }\left(1 \mathrm{st} \_\mathrm{H}\right)$, and the burst density at $5 \mathrm{~cm} \mathrm{~Pb}$ of the corresponding $\Gamma$-block, $n_{b}^{\max }(\Gamma 5 \mathrm{~cm} \mathrm{~Pb})$, (upper) and that between the maximum burst density at the 2nd hardon-block, $n_{b}^{\max }\left(2 \mathrm{nd} \_\mathrm{H}\right)$, and $n_{b}^{\max }(\Gamma 5 \mathrm{~cm} \mathrm{~Pb})$ (lower). The large red circles are for the "expected experimental data" of "PAMIR-XXI". Small crosses are for the simulated data of proton primaries and small circles are for iron primaries.

by the Chacaltaya burst detector, $n_{b}(\mathrm{Chac})$ and that by the 1st hadron-block, $n_{b}(1$ st_H $)$, and also the relationship between the burst density, $n_{b}(\mathrm{Chac})$, and that of the 2 nd hadron-block, $n_{b}\left(2 \mathrm{nd} \_\mathrm{H}\right)$, of the "PAMIR-XXI" detectors. The results are shown in Fig. 6. Using these relationships, we can transform the experimental data of the Chacaltaya hybrid experiment into "expected experimental data" for the "PAMIR-XXI" experiment. The fluctuation around the average relationships is rather large. Also some of the events possibly have no family of $\Sigma E_{\gamma} \geq 10 \mathrm{TeV}$ at Chacaltaya but give a large burst-density in the $\Gamma$-block at the Pamir experiment. These effects are not taken into account in the present analysis.

\subsection{Comparison of "expected experimental" data with simulations}

Figure 7 shows a correlation diagram between the maximum burst density at the 1 st hadron-block, $n_{b}^{\max }\left(1 \mathrm{st} \_\mathrm{H}\right)$, 

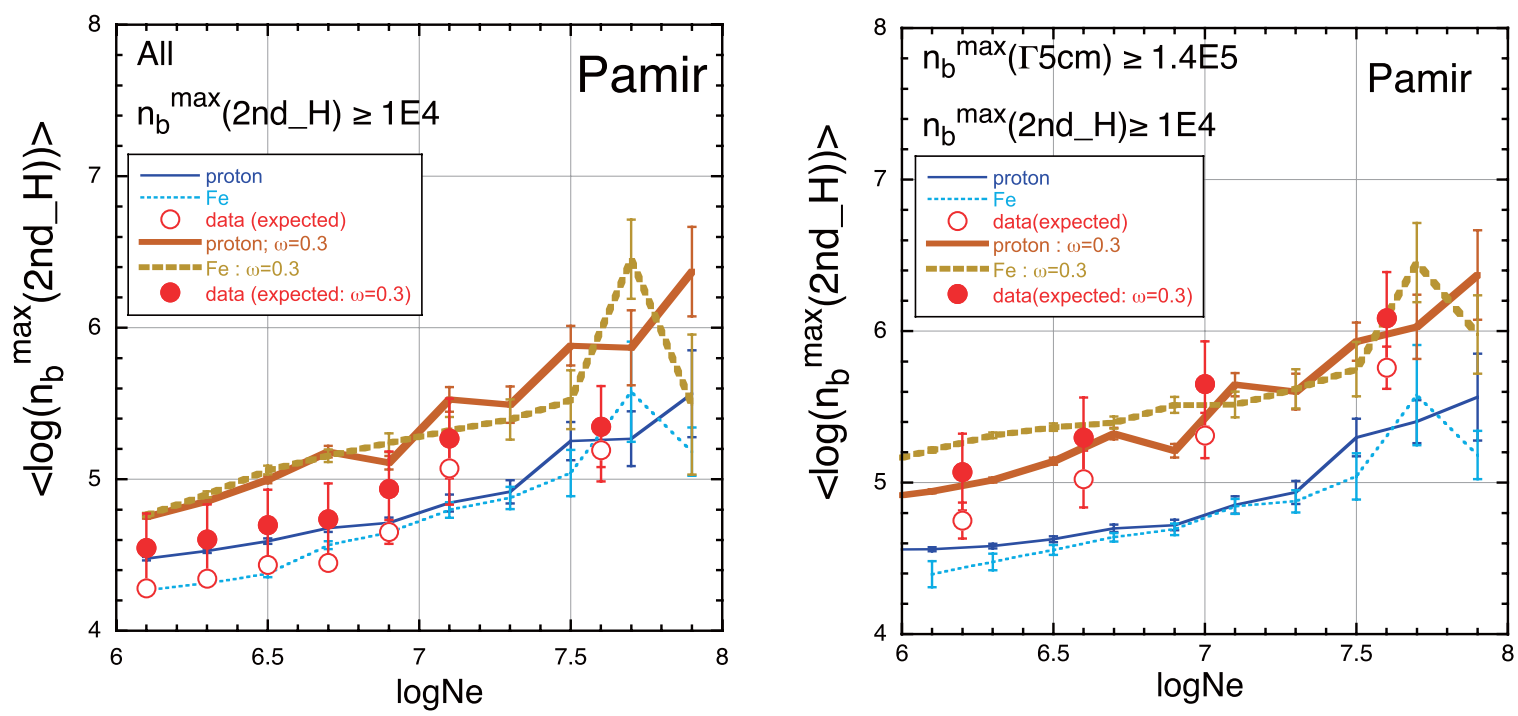

Figure 8. Shower size, $N_{e}$, dependence on the maximum burst density of the 2nd hadron-block, $n_{b}^{\max }\left(2 \mathrm{nd} \_\mathrm{H}\right)$, expected in the "PAMIRXXI" for all events with $n_{b}^{\max }\left(2 \mathrm{nd}_{-} \mathrm{H}\right) \geq 10^{4}$ (left) and for events with a large burst density at the $\Gamma$-block, $n_{b}^{\max }(\Gamma 5 \mathrm{~cm} \mathrm{~Pb}) \geq 1.4 \times 10^{5}$ (right). Open circles are those for "expected experimental data". Thin lines are those for the events simulated by CORSIKA/QGSJET01 with proton (solid) and Fe (dashed) primaries. Closed circles and thick lines are those in the case of introducing fluctuations in hadron-Air interactions in simulations with a parameter of relative fluctuation $\omega=0.3$.

and the burst density at $5 \mathrm{~cm} \mathrm{~Pb}$ in the corresponding $\Gamma$-block, $n_{b}^{\max }(\Gamma 5 \mathrm{~cm} \mathrm{~Pb})^{4}$, expected in the "PAMIR-XXI" experiment (see Fig. 3 for comparison with the Chacaltaya data). Also shown is that between the maximum burst density of the 2nd hadron-block, $n_{b}^{\max }\left(2 \mathrm{nd} \_\mathrm{H}\right)$, and $n_{b}^{\max }(\Gamma 5 \mathrm{~cm} \mathrm{~Pb})$ of $\Gamma$-block. We can see that a considerable number of "expected experimental" events accompany larger burst density in the hadron-block than do simulated events.

Figure 8 shows the shower-size, $N_{e}$, dependence on the maximum burst density of the 2nd hadronblock, $n_{b}^{\max }\left(2 \mathrm{nd} \_\mathrm{H}\right)$, in "PAMIR-XXI" for all events with $n_{b}^{\max }\left(2 \mathrm{nd} \_\mathrm{H}\right) \geq 10^{4}$ (upper) and for events with large burst density at the $\Gamma$-block, $n_{b}^{\max }(\Gamma 5 \mathrm{~cm} \mathrm{~Pb}) \geq 1.4 \times 10^{5}$ (lower), which correspond to events with family energy $\Sigma E_{\gamma} \geq 10 \mathrm{TeV}$ in the Chacaltaya hybrid experiment. If we analyze all the events together, the "expected experimental" data look like they are well described by the simulations, but if we pick up only the events with larger burst density in $\Gamma$-block, model calculations fail to describe the "expected experimental" data, i.e., the burst density in the hadron-block in those events is systematically larger than those of simulated events (see Fig. 2 for the comparison with the Chacaltaya data).

\subsection{Effect of fluctuations in hadron-Air interaction}

The possible existence of fluctuations in the cross-section of hadronic interaction is discussed in references $[14,15]$ and this fluctuation affects the attenuation of hadronic component in air-showers [15]. In the analysis of the Chacaltaya hybrid experiment, we have shown that the disagreement between experimental and simulation data becomes smaller when cross-section fluctuations are taken

\footnotetext{
4 The results using the burst-density $n_{b}^{\max }(\Gamma 4 \mathrm{~cm} \mathrm{~Pb})$ or $n_{b}^{\max }(\Gamma 6 \mathrm{~cm} \mathrm{~Pb})$ are not much different from those using $n_{b}^{\max }(\Gamma 5 \mathrm{~cm} \mathrm{~Pb})$.
}

into account [3]. Here we again introduce the fluctuation of the cross-section in high-energy hadron-air interactions and study the effect on the correlation between the burst density in the $\Gamma$-block and in the hadron-block in the "PAMIR-XXI" experiment. We apply the same procedure used in paper [15], that is, the cross-section, $\sigma(E)$, of hadron-air is sampled from the uniform distribution in the interval $\left[1-\sqrt{3 \omega} \sigma_{0}(E), 1+\sqrt{3 \omega} \sigma_{0}(E)\right]$. Here $\sigma_{0}(E)$ is the mean cross-section at energy, $E$, and $\omega$ is the relative fluctuation of the cross-section. We apply these modifications in the CORSIKA simulation code and calculate air-showers.

The results are shown in Fig. 8 for the case of $\omega=0.3$. The fluctuation of the cross-section affects the attenuation of the hadron component, then it also affects the "expected experimental" data. In the figure the closed circles are for the "expected experimental" data and thick lines for the simulated data taking into account the fluctuation of the cross-section. As seen in the figure, the "expected experimental" data are well described by model calculations including cross-section fluctuation especially for events with larger burst densities in the $\Gamma$-block.

\section{Summary and discussions}

We have shown that the unusual characteristics observed by the Chacaltaya hybrid experiment, in which the emulsion chamber data is one of the key ingredient of the analysis, can also be well seen in the "PAMIR-XXI" hybrid experiment, in which scintillator counters are used instead of emulsion chambers. If the "PAMIR-XXI" experiment is carried out as planned, statistics of the events increase $\sim 10$ times larger than those detected in the Chacaltaya hybrid experiment after $2 \sim 3$ years exposure. If the unusual characteristics, i.e., disagreement between experimental data and simulations, still remain in the "PAMIR-XXI" experiment, we must seriously consider the reason to explain it. As one of the possible reasons, we considered 
the effect of fluctuations in the cross-section of hadron-air interactions. It is found that the discrepancy between experimental and simulated data becomes smaller when the cross-section fluctuation is taken into account. Some changes of the characteristics of particle production may also cause the discrepancy between experimental and simulated data, e.g., recent results of the LHCf experiment show a more abundant neutron production rate to photon production than in the current model prediction in the very forward region in $\mathrm{p}-\mathrm{p}$ collisions at $\sqrt{s}=7 \mathrm{TeV}[16]$.

\section{References}

[1] N.Kawasumi et al., Phys. Rev. D 53 (1996) 3534

[2] C. Aguirre et al., Phys. Rev. D 62 (2000) 032003

[3] M. Tamada et al., Proc. 17th Int. Symp. on Very High Energy Cosmic Ray Interactions, Berlin (2012), EPJ Web of Conference 52 (2013) 07006

[4] Tibet AS $\gamma$ Collaboration (M.Amenomori et al.), Phys. Rev. D 62 (2000) 112002-1, 072007-3

[5] Tibet AS $\gamma$ Collaboration (M.Amenomori et al.), Phys. Lett. B 632 (2006) 58

[6] J. Blumer, R. Engel and J. R. Horandel, Prog. in Part. Nucl. Phys. 63 (2009) 58
[7] W.D. Apel et.al., Advances in Space Res. 53 (2014) 1456

[8] A.S. Borisov, K. Muminov, V. Galkin and V. Puchkov, Proc. 17th Int. Symp. on Very High Energy Cosmic Ray Interactions, Berlin (2012), EPJ Web of Conferences 52 (2013) 11002, Slide of the talk: https://indico.desy.de/conference OtherViews . py?view=standard\&conf $\mathrm{Id}=4594$

[9] J. Kempa, B. Pattison, E. Glaysz-Dziadus, L.W.Jones, R. Mukhamedshin, M. Tamada and Z. Wlodarczyk, Cent. Eur. J. Phys. 10 (2012) 723

[10] D. Heck, J. Knapp, J.N. Capdevielle, G. Schatz and T. Thouw, Report FZKA 6019 (1998), Fortshungzentrum Karlsruhe; available from https : / www. ikp. kit.edu/corsika/70.php

[11] N.N. Kalmykov, S.S. Ostapchenko and A.I. Pavlov Nucl. Phys. B, Proc. Suppl. 52B (1997) 17

[12] S. Agostinlli et al. Geant4 collaboration, Nucl. Inst. and Meth. A 506 (2003) 250

[13] T. Yamasaki and M. Tamada, Proceedings of 32th ICRC, Beijing (2011) Vol.1, p. 216

[14] B. Blättel et al., Phys. Rev. D 47 (1993) 2761

[15] G. Wilk and Z. Wlodarczyk, Phys. Rev. D 50 (1994) 2318

[16] O. Adriani et al. (LHCf Collaboration), Phys. Lett. B 750 (2015) 360 